\title{
Collective phase locked states in a chain of coupled chaotic oscillators
}

\author{
D. L. Valladares, ${ }^{1,3}$ S. Boccaletti, ${ }^{2,1}$ F. Feudel, ${ }^{4}$ and J. Kurths ${ }^{4}$ \\ ${ }^{1}$ Departamento de Fisica y Matematica Aplicada, Universidad de Navarra, Pamplona, Spain \\ ${ }^{2}$ Istituto Nazionale di Ottica Applicata, Largo E. Fermi, 6, I50125 Florence, Italy \\ ${ }^{3}$ Departamento de Física, Universidad Nacional de San Luis, Argentina \\ ${ }^{4}$ Institut fuer Physik, Universitaet Potsdam, PF 601553 Potsdam, Germany
}

(Received 25 October 2001; published 21 May 2002)

\begin{abstract}
We discuss the emergence of a collective phase locked state in an open chain of $N$ unidirectionally weakly coupled nonidentical chaotic oscillators. Such a regime is characterized by a Lyapunov spectrum where $N-1$ exponents that were zero in the uncoupled regime assume negative values as the coupling strength increases. The dynamics of such collective state is studied, and a comparison is drawn with the case of phase synchronization of a pair of coupled chaotic oscillators. In particular, it is shown that a full phase synchronized state cannot be constructed without at least partial correlation in the chaotic amplitudes.
\end{abstract}

DOI: 10.1103/PhysRevE.65.055208

PACS number(s): 05.45.Xt, 05.45.Jn

Synchronization of coupled chaotic oscillators has received a growing interest in the past years. Several types of synchronized motions have been studied, namely, complete or identical synchronization [1], generalized synchronization [2], phase locking (PL) [3], and lag synchronization [4]. While transitions among different types of synchronized regimes have been described [4], recently attempts to construct unifying formalisms encompassing these phenomena have been made [5].

In this paper, we describe the emergence of phase locked states in the collective behavior of a chain of weakly coupled chaotic oscillators, and we characterize the main features of such a behavior, discussing analogies and differences with the case of synchronized motions emerging in a pair of coupled chaotic oscillators.

PL of two periodic oscillators has been object of attention since the 17th century [6]. This phenomenon corresponds to the appearance of a given parametrically stable limit cycle within a torus. PL requires that any $2 \pi$ phase shift in one oscillator must be accompanied by a corresponding $(m / n) 2 \pi$ phase shift in the other $(m, n \in \mathcal{N})$. More recently, the above concept has been extended to that of phase synchronization of chaotic systems, describing a process through which a weak interaction between two chaotic oscillators having different rhythms mutually adjusts their phases in the course of the time, thus producing a collective dynamical state. Here, the locking condition was said to be $\mid \varphi_{1}$ $-(m / n) \varphi_{2} \mid<$ const $\left(\varphi_{1,2}\right.$ being the phases of the two oscillators), indicating a locking of the chaotic phases in the course of the time. Since its theoretical proposal [3], this phenomenon has been discovered to be ubiquitous in nature [7]. Furthermore, this phenomenon has been largely investigated in controlled laboratory experiments [8], as well as in infinite dimensional or space extended chaotic systems [9].

In the following, we will describe phase locked collective states in an open chain of unidirectional coupled chaotic oscillators. The system under study is composed of $N$ coupled nonidentical three dimensional Rössler oscillators [10], described by

$$
\dot{x}_{1}=-\omega_{1} y_{1}-z_{1}
$$

$$
\begin{gathered}
\dot{y}_{1}=\omega_{1} x_{1}+a y_{1}, \\
\dot{z}_{1}=f+z_{1}\left(x_{1}-c\right), \\
\dot{x}_{i}=-\omega_{i} y_{i}-z_{i}+\varepsilon\left(x_{i-1}-x_{i}\right), \\
\dot{y}_{i}=\omega_{i} x_{i}+a y_{i}, \\
\dot{z}_{i}=f+z_{i}\left(x_{i}-c\right),
\end{gathered}
$$

$i=2, \ldots, N$ representing the index of the oscillator. In Eqs. (1), $a=0.15, f=0.4$, and $c=8.5$ are fixed parameters, while the frequencies $\omega_{i}$ of the $N$ oscillators increase linearly by the rule

$$
\omega_{i}=\omega_{1}+(i-1) \frac{\omega_{N}-\omega_{1}}{N-1},
$$

where $\omega_{1}=0.985\left(\omega_{N}=1.0165\right)$ is the frequency of the first (the last) oscillator in the chain. Due to the unidirectional nature of the coupling, we will call $\omega_{1}$ the driving frequency of the chain. We notice that unidirectional coupling allows one to have a dominant frequency, with respect to which one can estimate synchronization features. Furthermore, unidirectional coupling schemes are suitable for investigating transient phenomena along the chain, which have relevance in some applied fields, such, e.g., neuroscience [11] and earth science [12]. In the following we will concentrate our study to the case $N=11$.

A convenient way to detect the emergence of phase synchronization phenomenon consists in monitoring the temporal evolution of phase differences among different oscillators $\left[\Delta \varphi_{i j}(t) \equiv\left|\varphi_{i}(t)-\varphi_{j}(t)\right|\right]$ and verifying that $\Delta \varphi_{i j}(t)$ fulfills the locking condition $\left|\Delta \varphi_{i j}\right|<$ const. The election of parameters of system 1 determines that the attractor, where the trajectory evolves, is phase coherent, and then it is possible to define the phase of each chaotic oscillator as $\varphi_{i}(t)$ $=\arctan \left[y_{i}(t) / x_{i}(t)\right]$ and the corresponding amplitude as $A_{i}(t) \equiv \sqrt{x_{i}^{2}(t)+y_{i}^{2}(t)}$. In order to detect a collective phase synchronization state in the chain, we have monitored the 

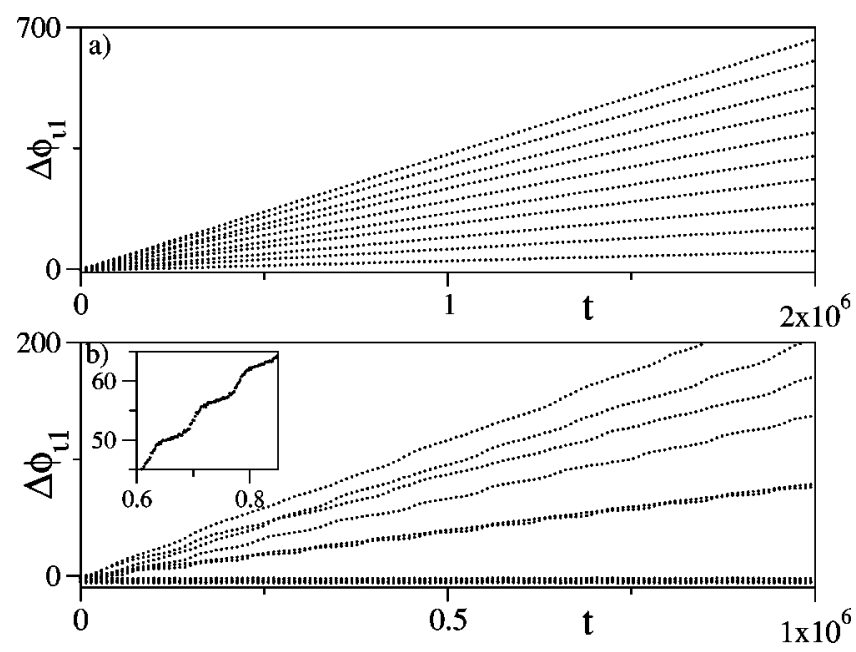

FIG. 1. Temporal evolution of the phase differences $\Delta \varphi_{i 1}$ $\equiv\left|\varphi_{i}(t)-\varphi_{1}(t)\right|$ at $\varepsilon=0.0015$ (a) and at $\varepsilon=0.015$ (b). $i$ $=2, \ldots, 11$ increases from bottom to top curve in the figure. The inset of (b) highlights the $2 \pi$ phase jumps in the evolution of $\Delta \varphi_{61}$.

phase difference between each coupled oscillator and the first one $\Delta \varphi_{i 1}(t)$ at different coupling values $\varepsilon$.

At very small coupling strengths, all oscillators evolve in an unsynchronized manner, as is demonstrated in Fig. 1(a), where we report the phase differences $\Delta \varphi_{i 1}$ vs time for $\varepsilon$ $=0.0015$. The observed phase difference evolutions align quasiperfectly on straight lines whose slopes correspond to the mean frequency differences $\Delta \omega_{i 1} \equiv\left|\left\langle\omega_{i}\right\rangle-\left\langle\omega_{1}\right\rangle\right|$. As a consequence, each chaotic oscillator evolves with a different rhythm, and no phase locking is produced.

As the coupling increases, system (1) experiences a transition toward a collective state, wherein some oscillators $(i$ $=2,3,4,5)$ display phase locking with the drive frequency $\omega_{1}$, whereas all the other oscillators evolve in a phase unsynchronized regime [Fig. 1(b), for $\varepsilon=0.015$ ]. Notice that here the oscillator $i=6$ is located at the borderline of phase synchronization, displaying $2 \pi$ jumps between successive plateaus of constant phase difference [inset of Fig. 1(b)]. The appearance of $2 \pi$ phase slips at the transition between an unsynchronized and a phase locking regime was indeed considered as a speciality of phase synchronized phenomenon [3].

In order to give evidence of PL in our chain, we performed a long simulation trial, in which the system was prepared in the unsynchronized regime $(\varepsilon=0.0015)$. The results are shown in Fig. 2. Initially, all oscillators evolve in a phase unsynchronized manner (left part of the curves). At $t$ $=5000$ time units, a sudden change in the coupling value is realized, and we set $\varepsilon=0.06$ in system (1). The effect is that, after a very short transient time, all oscillators begin evolving in a phase locked regime, and consequently all phase differences converge to constant values (right part of the curves). This indicates that the dynamics of system (1) could support a global PL regime for a sufficiently high coupling value.

Another independent check of the above is to monitor the differences in the mean frequency $\Delta \omega_{i 1} \equiv\left|\left\langle\omega_{i}\right\rangle-\left\langle\omega_{1}\right\rangle\right|(i$

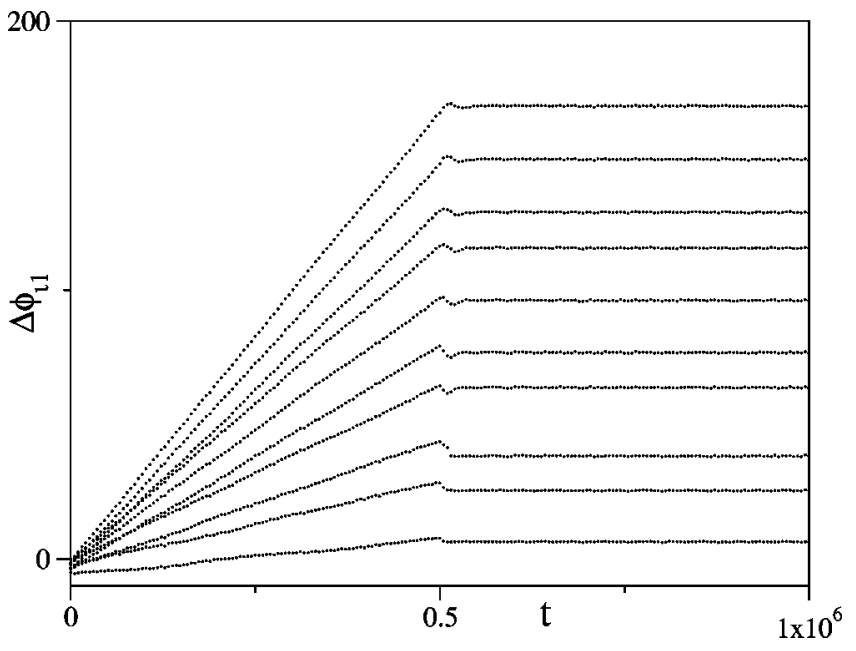

FIG. 2. Temporal evolution of the phase differences $\Delta \varphi_{i 1}$ $\equiv\left|\varphi_{i}(t)-\varphi_{1}(t)\right| . i=2, \ldots, 11$ increases from bottom to top curve in the figure. Initially, the system is prepared in the unsynchronized regime $(\varepsilon=0.0015)$. At $t=5000$ the coupling is changed to $\varepsilon$ $=0.06$. As a result, all oscillators mutually lock their phases.

$=2, \ldots, 11)$ as functions of the coupling strength $\varepsilon$. The results are shown in Fig. 3. $\left\langle\omega_{i}\right\rangle$ are obtained as time averages (over periods much larger than the oscillating periods) of the derivatives of the instantaneous phases $\varphi_{i}(t)$. Figure 3 exhibits clearly that the process through which the system reaches a completely PL state consists in phase locking processes between successive oscillators in the chain and the drive oscillator. Such phase lockings occur at increasing $\varepsilon$ values, indicating that one can inspect different dynamical regimes in our chain, namely, a phase unsynchronized regime $(\varepsilon<0.0075)$, a partial PL regime characterized by an increasing number of phase locked oscillators $(0.0075<\varepsilon$ $<0.052$ ), and a full PL regime for $\varepsilon>0.052$, characterized by a collective evolution of the instantaneous phases of all oscillators in the chain.

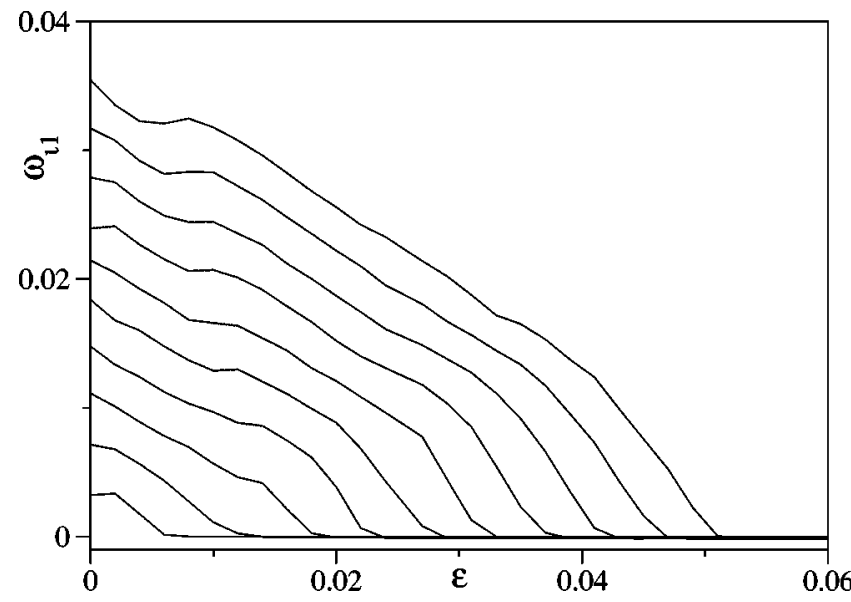

FIG. 3. Difference in the mean frequency $\Delta \omega_{i 1} \equiv\left|\left\langle\omega_{i}\right\rangle-\left\langle\omega_{1}\right\rangle\right|$ of the chaotic oscillators (vertical axis) vs the coupling strength $\varepsilon$ (horizontal axis). The process through which the system reaches a completely phase synchronized state is realized via successive locking processes of the oscillators' phases to the drive phase. 


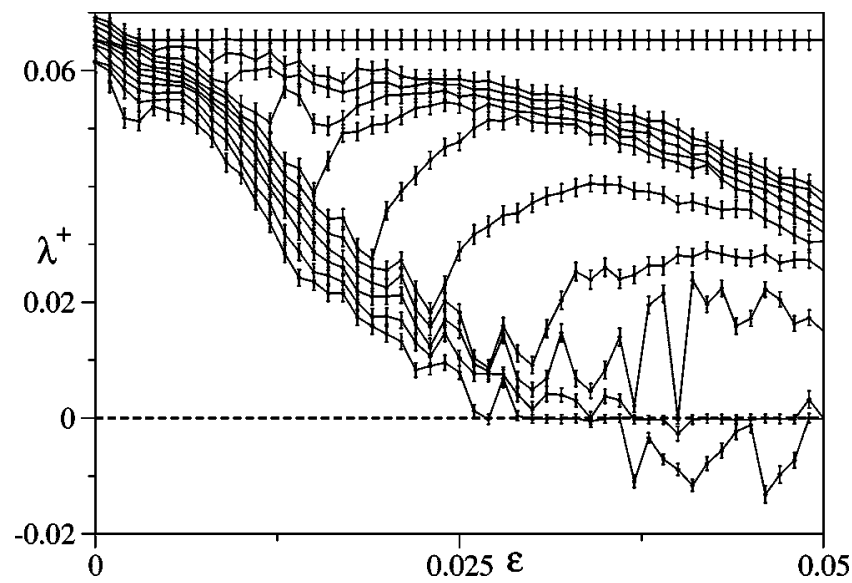

FIG. 4. Lyapunov exponents in the spectrum vs the coupling strength $\varepsilon$. First largest 11 Lyapunov exponents in the spectrum. The error bars account for the residual fluctuations in the values of the corresponding Lyapunov exponents.

Next we characterize more quantitatively the observed synchronization scenario. In a pair of coupled chaotic oscillators it is possible to determine the transition to a PL regime studying the changes of Lyapunov spectrum as a function of the coupling. This regime appears at coupling values wherein a zero exponent passes to a negative value at the same time at which all exponents that were positive at no coupling remain positive [3]. The above fact indicates that the system has globally adjusted the phases of the oscillators, while almost no correlation in the amplitudes is built. In the present case, the Lyapunov spectrum at $\varepsilon=0$ is constituted by $N$ positive, $N$ zero, and $N$ negative exponents, where the $N$ zero exponents are associated with the phases of the chaotic oscillators.

Figures 4, 5, and 6 show how the Lyapunov spectrum is modified by increasing the coupling strength. Lyapunov exponents have been calculated over a running time of about 55000 oscillations of the systems, and error bars account for

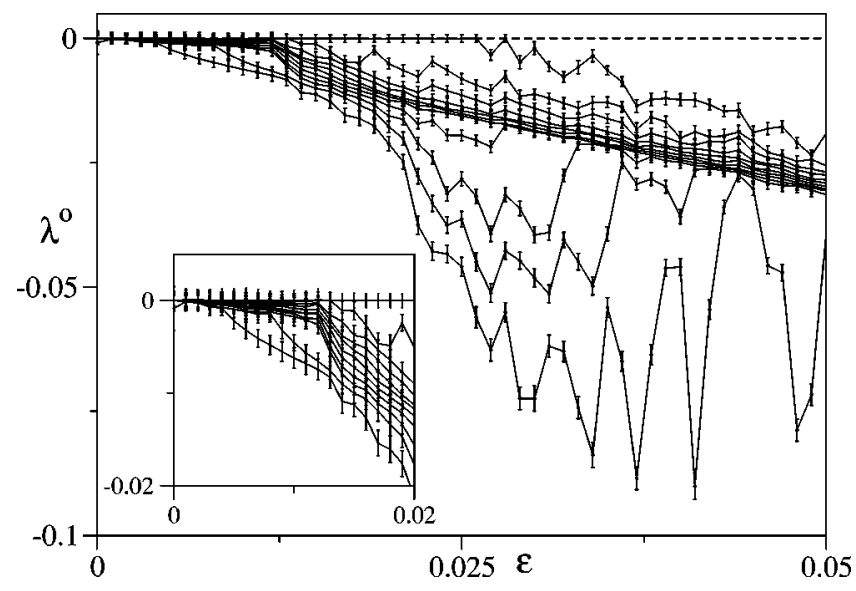

FIG. 5. Lyapunov exponents in the spectrum vs the coupling strength $\varepsilon$. Second largest 11 Lyapunov exponents in the spectrum. The inset shows a zoom in the range $0<\varepsilon<0.02$. The error bars account for the residual fluctuations in the values of the corresponding Lyapunov exponents.

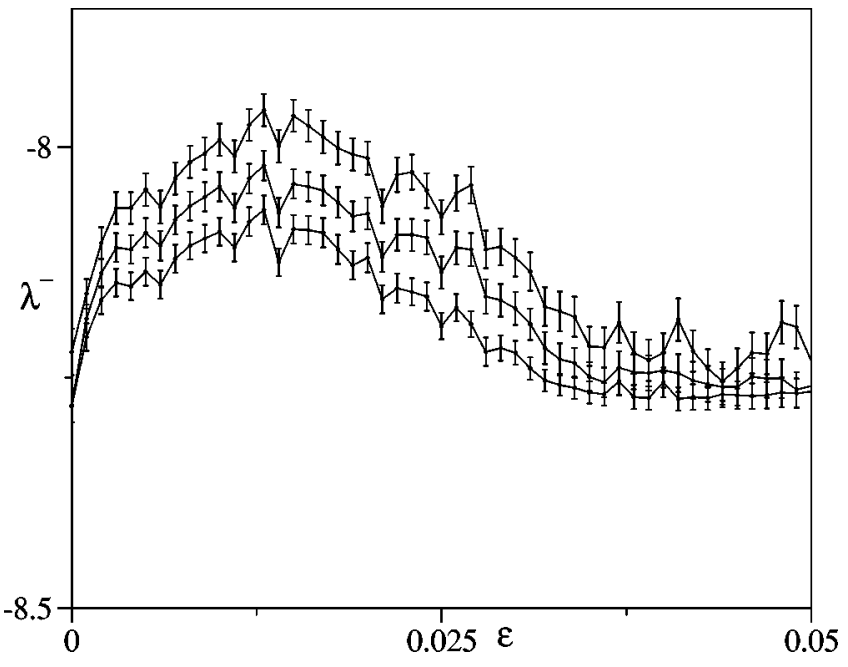

FIG. 6. Lyapunov exponents in the spectrum vs the coupling strength $\varepsilon$. The three largest negative exponents in the Lyapunov spectrum (23rd to 25th). The error bars account for the residual fluctuations in the values of the corresponding Lyapunov exponents.

residual fluctuations in the Lyapunov exponents at that time. In the uncoupled case $(\varepsilon=0)$, the Lyapunov spectrum is constituted by $N=11$ positive exponents $\left(\lambda^{+}\right), N$ zero exponents $\left(\lambda^{0}\right)$, and $N$ negative exponents $\left(\lambda^{-}\right)$. Figure 4 reports the modification in the values of the largest $N$ exponents in the spectrum, as the coupling strength increases. There, one clearly sees that all $N$ exponents remain positive in the range $0<\varepsilon \lesssim 0.027$. At larger couplings, one exponent passes to a negative value. This implies that no amplitude correlations are built within the system for $\varepsilon \leqq 0.027$.

In Fig. 5 we report the values of $\lambda^{0}$ (exponents that were 0 in the uncoupled case), as the coupling strength increases. One can easily appreciate the fact that successive exponents pass from zero to a negative value, as $\varepsilon$ increases, until $\varepsilon$ $\simeq 0.027$. From the considered coupling scheme, the first oscillator comes out to be uncoupled, therefore we expect to have a positive Lyapunov exponent in the spectrum that is insensitive to $\varepsilon$ (see Fig. 4), as well as a zero Lyapunov exponent that finally represents the dominant phase imposed by the driving oscillator. This is an important fact that indicates the presence of our system in a regime $(0<\varepsilon$ $<0.027)$ where successive oscillators lock their phases with the drive phase without building appreciable correlations in the corresponding chaotic amplitudes. In the inset, a zoom is shown in the range $0<\varepsilon<0.02$, with the purpose of highlighting how the zero Lyapunov exponents in the spectrum passes sequentially to negative values.

In Fig. 6 we report the values of the three largest negative exponents in the Lyapunov spectrum, showing that the corresponding values are not changed in sign, as the coupling increases.

The behavior of the Lyapunov spectrum helps us to understand quantitatively the scenario of synchronization emerging in our system. For sufficiently small coupling strength, the oscillators evolve in an unsynchronized way, (see Fig. 1 for $\varepsilon=0.0015$ ). At intermediate coupling strengths (see Fig. 1 for $\varepsilon=0.015$ ), the system displays again 
$N$ positive Lyapunov exponents in the spectrum, whereas some of the exponents that were originally zero have passed to negative values (see Fig. 5). As a result, the collective dynamics of system (1) displays no correlation in the chaotic amplitudes of the oscillators, but partial locking in the phases.

Finally at large coupling strength, the system evolves in a complete PL regime (Fig. 2 at $\varepsilon=0.06$ ). We recall that a direct cross check in the coupling values between Fig. 3 and Fig. 5 is not possible, since it has been shown that a stable PL regime sets in for coupling strengths slightly larger than the ones at which a Lyapunov exponent passes from zero to a negative value [3].

However, by comparing Fig. 3 with Figs. 4 and 5, it is easy to understand that system (1) cannot realize a completely PL state without having at least partial correlation in the amplitudes. This is due to the fact that the $\varepsilon$ value at which the last oscillator locks its phase with the drive $(\varepsilon$ $\simeq 0.052$ in Fig. 3) corresponds to a situation where at least one originally positive Lyapunov exponent has passed to a negative value (see Fig. 4), thus indicating the settings of amplitude correlation in at least a pair of chaotic oscillators in the chain. This fact differentiates our observation from the scenario of synchronization phenomena occurring in a pair of chaotic oscillators. In this latter case, indeed, either complete phase synchronization appears before the settings of amplitude correlation, or a direct transition to complete or generalized synchronization takes place without any intermediate phase locking regime. At variance, in the case studied here, a complete phase locked regime cannot be realized over the whole chain, without implying at least partial correlation in the chaotic amplitudes. On this basis, we highlight that the above limit for phase synchronization in a chain of chaotic units represents a counterintuitive effect that could not have been predicted on the basis of the known results on phase synchronization in pairs of coupled chaotic oscillators. We finally remark that the observed scenario has been studied for a purely unidirectional coupling scheme. This leaves open the interesting problem of to what extent the phenomena described here can emerge in chains of bidirectionally but asymmetrically coupled chaotic units, which can be the case in many practical situations.

The authors acknowledge many fruitful and illuminating discussions with F. T. Arecchi, K. Josic, D. Maza, A. Pikovsky, A. Politi, M. G. Rosenblum, and J. W. Shuai that led to the ideas discussed in this Rapid Communication. Work was partly supported by the European Contract No. HPRNCT-2000-00158.
[1] H. Fujisaka and T. Yamada, Prog. Theor. Phys. 69, 32 (1983); L.M. Pecora and T.L. Carroll, Phys. Rev. Lett. 64, 821 (1990).

[2] N.F. Rulkov, M.M. Sushchik, L.S. Tsimring, and H.D.I. Abarbanel, Phys. Rev. E 51, 980 (1995); L. Kocarev and U. Parlitz, Phys. Rev. Lett. 76, 1816 (1996).

[3] M.G. Rosenblum, A.S. Pikovsky, and J. Kurths, Phys. Rev. Lett. 76, 1804 (1996).

[4] M.G. Rosenblum, A.S. Pikovsky, and J. Kurths, Phys. Rev. Lett. 78, 4193 (1997); S. Boccaletti and D.L. Valladares, Phys. Rev. E 62, 7497 (2000).

[5] I.I. Blekhman, A.L. Fradkov, H. Nijmeijer, and A.Yu. Pogromsky, Syst. Control Lett. 31, 299 (1997); R. Brown and L. Kocarev, Chaos 10, 344 (2000); S. Boccaletti, Louis M. Pecora, and A. Pelaez, Phys. Rev. E 63, 066219 (2001).

[6] C. Hugenii, Horoloquium Oscilatorium (Parisiis, France, 1673).

[7] C. Schafer, M.G. Rosenblum, J. Kurths, and H.H. Abel, Nature (London) 392, 239 (1998); P. Tass, M.G. Rosenblum, J. Weule, J. Kurths, A. Pikovsky, J. Volkmann, A. Schnitzler, and H.J. Freund, Phys. Rev. Lett. 81, 3291 (1998); A. Neiman, X. Pei, D. Russell, W. Wojtenek, L. Wilkens, F. Moss, H.A. Braun,
M.T. Huber, and K. Voigt, ibid. 82, 660 (1999); B. Blasius, A. Huppert, and L. Stone, Nature (London) 399, 354 (1999).

[8] C.M. Ticos, E. Rosa, Jr., W.B. Pardo, J.A. Walkenstein, and M. Monti, Phys. Rev. Lett. 85, 2929 (2000); D. Maza, A. Vallone, H. Mancini, and S. Boccaletti, ibid. 85, 5567 (2000); E. Allaria, F. T Arecchi, A. Di Garbo, and R. Meucci, ibid. 86, 791 (2001); W. Wang, I.Z. Kiss, and J.L. Hudson, ibid. 86, 4954 (2001); D.J. DeShazer, R. Breban, E. Ott, and R. Roy, ibid. 87, 044101 (2001).

[9] S. Boccaletti, J. Bragard, F.T. Arecchi, and H. Mancini, Phys. Rev. Lett. 83, 536 (1999); H. Chaté, A. Pikovsky, and O. Rudzick, Physica D 131, 17 (1999); S. Boccaletti, D.L. Valladares, J. Kurths, D. Maza, and H. Mancini, Phys. Rev. E 61, 3712 (2000).

[10] O.E. Rössler, Phys. Lett. 57A, 397 (1976).

[11] Z.F. Mainen and T.J. Sejnowski, Science 268, 9 (1995); W. Singer, Neuron 24, 49 (1999).

[12] see, e.g., D. L. Turcotte, Fractals and Chaos in Geology and Geophysics (Cambridge University Press, Cambridge, England, 1997). 